\title{
Contribution to Development of Modular Compliant Anthropomorphic Robot Hand
}

\author{
A. Rodić ${ }^{1}$, B. Miloradović ${ }^{1}$, S. Popić ${ }^{1}$, S. Spasojević ${ }^{1}$ and B. Karan ${ }^{2}$ \\ ${ }^{1}$ University of Belgrade, Institute MihajloPupin, Serbia, e-mail: \\ aleksandar.rodic@pupin.rs \\ ${ }^{2}$ Technical Institute of Serbian Academy of Science, Serbia, e-mail: \\ branko.karan@itn.sanu.ac.rs
}

\begin{abstract}
The paper is addressed to development of modular compliant robotic hand characterized by anthropomorphic structure and functionality. The prototype is built based on experience of development other contemporary artificial hands and taking into account complementary aspects of human hand bio-mechanics. The robot hand developed in the Institute Mihailo Pupin was called the "Pupin hand". Its purpose regards to research as well as for possible implementation with service and medical robot devices as the advance robot end-effector. The paper considers mechanical design, system identification, modeling and simulation and also acquisition of biological grasping skill taken from humans. The mechanical structure of the tendon-driven, multi-finger, 23 degrees of freedom compliant robot hand is presented in the paper. Corresponding model of the hand is represented by corresponding multi-body rigid system with complementary structural elasticity. Some characteristic simulation results are given in the paper in order to validate the design concept applied. For the purpose of experiments with motion capture of human grasping skill by the hand, appropriate experimental setup including infrared Kinect sensor is applied. The aim of use the Kinect sensor is to acquire human skill of grasping and map it to the artificial device. Future directions of research and development are given in the conclusion, too.
\end{abstract}

Key words: Anthropomorphic modular hand, tendon-driven hand, compliant hand, Kinect sensor

\section{Introduction}

The hand is rather complex limb of human body. It has a wide capability in object manipulation and grasping. One uses hands in different tasks like holding, shaking, grasping, touching, etc. Because of that, it is very difficult to design a robotic hand that is able to perform all of these tasks in a human manner. Artificial, i.e. robotic hand could be a part of industrial, service or medical robot. However, also can be a part of human body as an advanced prosthetic device. That means design of the artificial hand depends on its purpose. 
Bio-inspired artificial hand is actually universal gripper capable to optimally manipulate with objects of different sizes and shapes. Back in 1919 Schlesinger [1] has defined six basic ways of grasping as presented in Fig. 1. In order to achieve these movements it is necessary for finger parts to have some kind of actuation. For that matter, several types of actuators can be used: servomotors, pneumatic or hydraulic cylinders (artificial muscles) as well as some more advanced "motors" such as piezoelectric, memory alloy, etc. In this paper, mechanical design is considered, while the control will be reviewed in the following one [2].

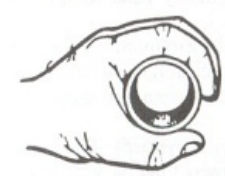

CYLINDRICAL GRASP

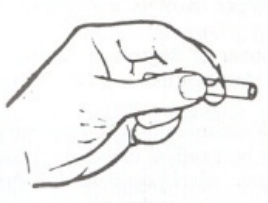

PALMAR

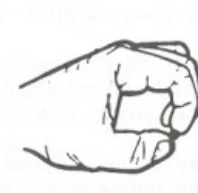

TIP

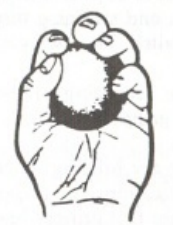

SPHERICAL GRASP

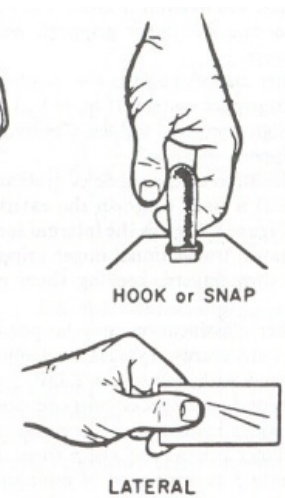

Fig. 1 The various types of prehension.

\section{State-of-the-Art}

More than 30 years development of artificial hand has been the subject of interest in many research centers. Many studies have been made and a large number of research papers have been published in which contributors give advantage to different types of designs. Reference [3] shows that serious approach to this subject began in early eighties at Stanford (JPL) and Massachusetts (MIT) Universities.

However, we cannot forget the fact that, in mid sixties, in Belgrade at Mihailo Pupin Institute was made "The Belgrade hand", device that is described in the majority of the text-books of Robotics [4]. Even though it was actuated with only one geared motor, complex lever mechanism had enabled contraction and protrusion of fingers. Force sensors, situated in finger tips, controlled power supply during the contact with environment Fig. 2.

Although one of the conclusions in [3] was that compliance cannot be achieved with simple design, the elegant solution with elastic connections gave system a limited compliance. If we wish to copy human anatomy, we need the actuation for every joint in robotic hand. Since every finger has 3 joints of which metacarpal (MCP) has 2 degrees of freedom (DOF), it means that the whole hand has at least 
20 DOFs. This large number of joints would imply the use of large number of actuators. From the mechanical point of view, actuators could be placed in the joints, near the joints or far away from the joints (Figs. 4 and 5). All of these approaches have its advantages as well as disadvantages [5], [6].
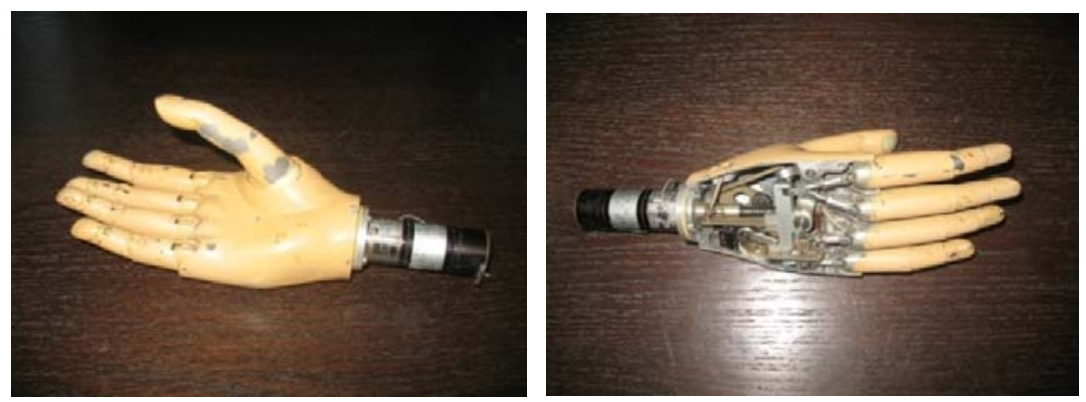

Fig. 3 The 'Belgrade hand'. The leverage mechanism that produces synergy of finger motions.

For example, in design where motors are not inserted near joints, a driving force is transferred via system of levers or using cables - tendons. This setup is also known in industrial robot design for the purpose of better mass distribution, but it also has lot of disadvantages such as additional elasticity, friction, nonlinearity etc. On the other hand, the motors integrated in the joints or close to them have as result significant increasing costs due to producing new types of smaller mechanical components like gearboxes, servomotors, encoders, force sensors, etc. In [7], the motors built in the DLR/HIT Hand-I (Fig. 6) have diameter of $20 \mathrm{~mm}$ and height of $10 \mathrm{~mm}$ and consequently have the weight of only 15 grams. They are paired with the similarly sized gearboxes. Transmission of torque to the joint is accomplished with miniature toothed belts. Example of joint actuation by $2 \mathrm{~N}$ or $\mathrm{N}+1$ tendons can be seen in Fig. 5.

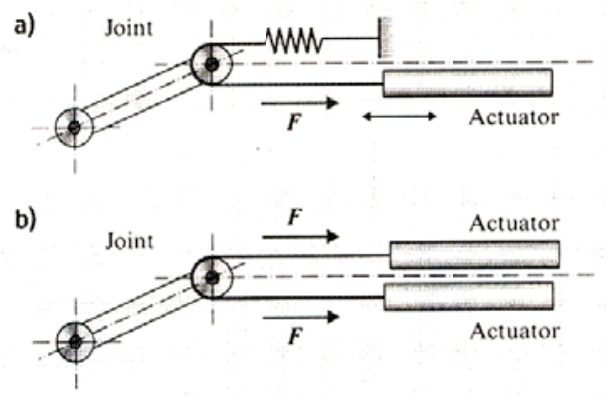

Fig. 4 a) single acting actuator with an antagonist passive element (spring), b) an agonist - antagonist configuration 


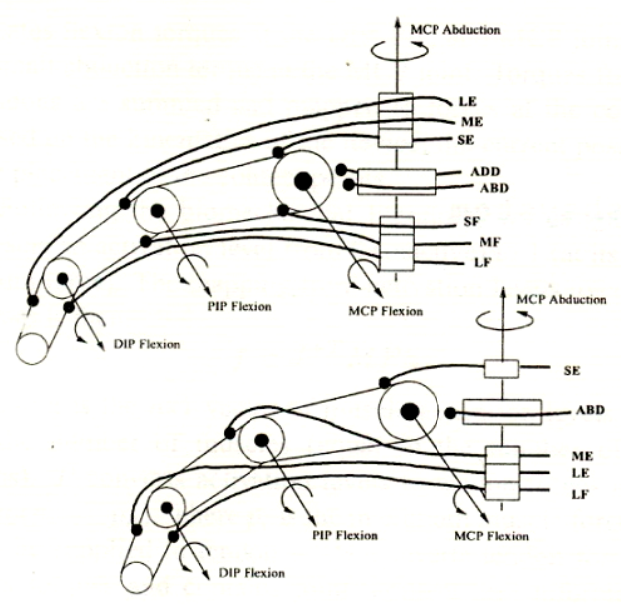

Fig. $52 \mathrm{~N}$ and $\mathrm{N}+1$ tendon-driven robot fingers where $\mathrm{N}$ is the number of DoFs (e.g. DoF=4)
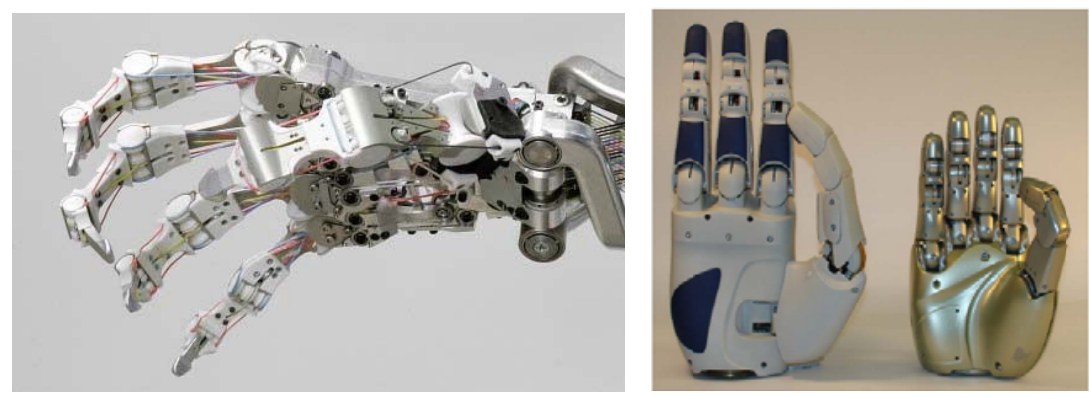

Fig. 6 The advanced DLR hand driven by tendons (left). DLR/HIT hands (right).

German Institute DLR in the past 10 years has significant results in this area. Few different types of robotic hands have been designed (Fig. 6). In [8] are also analyzed some of designs mentioned above with similar conclusions. Either price is too high or performances are not at satisfactory level or simply they are not commercialized yet.

Design of anthropomorphic robot hand capable to perform a lot of these capabilities on human manner requires large number actuators, i.e. DoFs. There are few possibilities to convey power from actuator to the joint. First one is miniaturization of motors and reducers (gearboxes). This approach is very expensive because it requires additional development a small components that could be installed in or close to joints. These hands are very complex, heavy, and needs large energy consumption. Hands with tendon driven fingers have advantages in ability 
to use relatively large space in forearm for location of motors but problem lies in complexity of task in which way pass the tendon through the wrist, palm up to phalanges. Use of conduit tubes reducing the design complexity but it introduce additional problems due to more friction that requires good maintenance as well as easy tendon replacement.

Between many others, the Shadow hand [9], [10] is a contemporary hand available in the market and based on pneumatic actuation (artificial muscles). It has 24 DoFs which produce power close to humans but velocity is approximately reduced by half. In its design the different modern materials are used. To produce all the movements this hand has 40 Mckibben artificial muscles and additional number of valves and all of them are integrated in the forearm.

A modular structure of robot hand enables easier assembling (integration) of the system as well as easier maintenance. In the same time, finger modules can be combined in different way to vary the hand configuration..

\section{Mechanical design}

The main objective of this project regards to design of a modular compliant anthropomorphic robot hand. The mechanical design of the hand has to ensure the device to looks like a human hand and to achieve similar functionalities as the biological model. Based on these assumptions, the choice was made to develop a modular tendon-based compliant artificial hand. For this purpose, the high-torque $\mathrm{RC}$ motors are integrated in the forearm link together with the power and control electronics. The power supply is not included in the device and it is left to be out of the system in order to decrease the overall mass. With new versions of robot hand, it is planned that the RC motors should be replaced by the corresponding miniature, powerful, but more expensive actuators in perspective. Solutions based on pneumatic or hydraulic actuation (artificial muscles) were discarded because of noise during operation and potential problems with control of fluid pressure. leakage, etc.

The Pupin hand is designed to be modular (five independent finger modules are assembled into a unique system) and made of light composite materials (Fig. 7). However, in the first phase, functional check is verified on 3D printer. All fingers have the same shape and design, and the length will be adjusted by position of the finger. At the bottom of each finger, where the fingers connect to the palm, additional reinforcements are done. There is an original connection between the lower segments which is realized by use of strong composite glue, few millimeters thick layer. That gives the hand certain passive compliance and elasticity related to the vertical axis, and it is manifested during grasping of the objects that lie in the palm. The phalanges are connected by low-ductile tendons through the pulley (Fig. 7). Every finger has 4 DoFs in mechanical sense. This implies that all of 20 cables (strings) should be implemented and connected to RC motors that are packed in the 
forearm in two rows arranged in a cascade wing with specially designed lowfriction cable guides. In such a way, each motor is shifted for the value that corresponds to the thickness of the tendon. So, tendons cannot come to collision and mutual friction.

To ensure compliance, as well as shock prevention to the driving (power transmission) system, the additional springs are integrated with tendons. Two design possibilities are considered as possible. The first one assumes torsion springs to be inserted in between the phalanges. The second one puts the spring in the line with tendons and this solution is assumed as the first approach. Regardless to the specific side effects, this contributes to a better tendon behavior, primarily in terms of disturbances as well as while grasping the objects of variable shape. All tendons from the fingers are collected behind the wrist joint of the hand and forwarded towards the motors. At the fingertips, corresponding contact sensors are planned to be attached, while other system sensors will be considered later in accordance with other technical requirements [6], [11], [12].

In order to reduce the cost of production, the first finger prototypes are built using 3D printer. This way of production is commonly used in rapid prototyping processes. Contact surfaces are planned to be covered with soft artificial materials (like synthetic rubber or polyurethane) with the aim of creating the human skin qualities as more as possible. This rapid method provided us in a short time with useful information relating primarily on the method, how a tendon can be attached to finger parts and how to conduct it along the finger to the motor. Tests were made by using fishing chord made of microfiber. However, our intention was to acquire and use more reliable material such as so called Dyneema material, frequently used in such kind of projects.

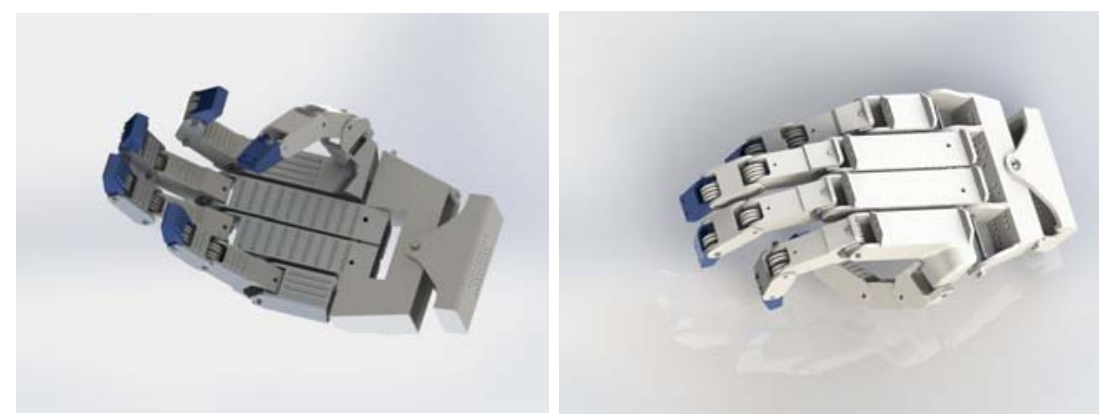

Fig. 7 CAD model of the "Pupin hand" developed. The bottom side (left) and the top side (right)

In [13] it is analyzed that this material is chosen over the steel cables, Kevlar or Aramid. Steel is too heavy and susceptible to constant and interchangeable bending loads, has more friction from the others and also has a destructive effect on the surface of softer materials in direct contact with them. On the other hand, Kevlar and Aramid have much better properties in relation to the friction and deformation. Their termination is complicated since knots weaken the tendon strength sig- 
nificantly. Compared to before mentioned materials, Dyneema has the best performances, the lower friction and termination can be done accurately by splicing. Tendons made from Dyneema have easy implementation and maintenance. Also, Dyneema has a good vibration characteristics, excellent abrasion and fatigue resistance as well as chemical and temperature persistency [14].

\section{Modeling and simulation}

Human hand, in mechanical sense, represents a multi-body rigid system with elastic joints and ductile power transmission (tendons). The hand in this project is modeled as a branched mechanism with 23 DoFs amongst are the following ones: five finger chains along with 4 DoFs every, 2 DoFs for yaw and pitch of hand in the wrist joint, and single twist-joint of the forearm (Fig. 8). Elasticity of the robot hand arises from tendons as well as from elastic joints. The elastic joints are marked in Fig. 8 by the "deflection" and "extension" titles that regards to the type of passive joints. The elastic components in the hand structure potentially worse controllability of the system but improve its compliance and ability of adaptation to system overloading. Due to the fore mentioned antagonistic effects of system elasticity, the accurate modeling of the entire robot hand is of great interest for control design of the device.

The model of rigid body dynamic of the Pupin hand is defined by corresponding differential equations in vector form. The equations of motions are defined in accordance with [15]. The following assumption is made: no inertial coupling between the motors and the joints, being the motors are dislocated into the forearm according to the mechanical design. The model of the hand is defined in the following way:

$$
M(q) \ddot{q}+C(q, \dot{q}) \dot{q}+G(q)+\tau_{\mu}(q)=\tau(q)+\tau_{c}+J(q) F
$$

where $M(q)$ is the system inertia matrix, $C(q, \dot{q}) \dot{q}$ are Coriolis and centrifugal terms, $\tau_{\mu}$ regards to the joint friction torques (due to tendon passing over the joint pulleys) and $G(q)$ is vector of gravity torques. Right side members are: the joint torques $\tau(q)$ and the external payload torques which are exhibited by the environment and defined by the term $J(q) F$, where $J(q)$ is the Jacobian matrix of the hand. The compliant torques, derived from the spring forces, are defined by the term $\tau_{c}$. The (1) can be extended by inserting (2):

$$
\tau(q)=P f_{t}
$$



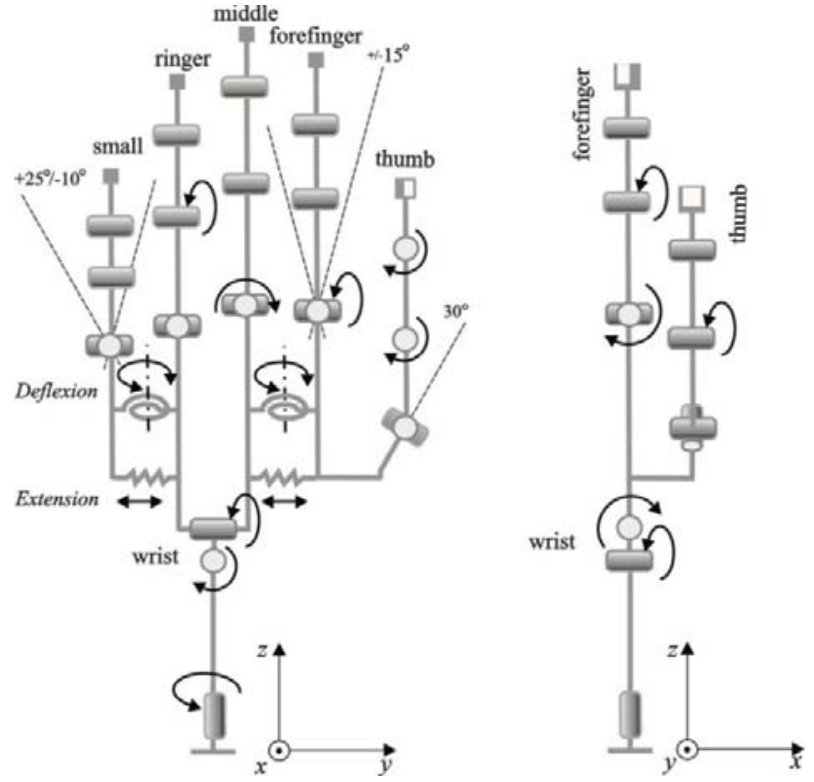

Fig. 8 Kinematic scheme of the 23 DoFs mechanism of the "Pupin hand".

where $\mathrm{P}$ (the coupling matrix) has full row rank and $f_{t}$ is the pulling constraint for the tendon forces.

The dynamics of the actuators (RC motors) applied with robot hand can be defined by vector equation:

$$
B \ddot{\theta}+\tau_{\theta, f}+E f_{t}=\tau_{m}
$$

where $\ddot{\theta}$ is motor angular acceleration, $B$ is the motor inertia matrix, $\tau_{\theta, f}$ are motor and tendon friction, $E f_{t}$ are torques produced by the tendon forces and $\tau_{m}$ are the motor torques. In this phase of development, the Futaba S3305 high-torque servo motor with metal gears is chosen because the project requires high torque from a standard size servo motor. The motor parameters are given in Tab. 1.

Table 1. Parameters of the Futaba S3305 RC motor.

\begin{tabular}{cccc}
\hline Speed $(\mathrm{rad} / \mathrm{s})$ & Torque $(\mathrm{Nm})$ & Size $(\mathrm{mm})$ & Weight $(\mathrm{g})$ \\
\hline 5.00 & 0.89 & $49.5 \times 20 \times 38$ & 46.5 \\
\hline
\end{tabular}

Assuming that the winding drum (winch) has a diameter of $10 \mathrm{~mm}$ then it enables the radial force of $89 \mathrm{~N}$ according to the Tab. 1. As part of that force is lost due to the friction resistance, the desired $30 \mathrm{~N}$ force per finger is still ensured. 
The model of the particular hand finger with four low-tensile and low-friction tendons to transmit the torques from motors to the particular finger joints is presented in Fig. 9. The tendons $i=1, \ldots, 4$ are driven by four winches rotating at $\omega_{i}$ r.p.m. The tendons $i=2,3,4$ are laid upon the corresponding pulleys in such a way to enable appropriate rotation of the corresponding joints while the tendon $i=1$ is simply placed over the wrist joint axle (Figs. 9a and 9b). Corresponding springs are inserted in the system to ensure desired compliance of the device, too. The springs are characterized by the stiffness coeficients $k_{i}$. Springs serve in the system to amortize destructive effects of the excessive payload that can be produced at robot finger in certain cases. Displacements $\delta_{i}$ and $\delta^{\prime}{ }_{i}$ shown in Fig. 9 are corresponding spring extensions. The particular tendons terminate in the points $E_{0}, \cdots, E_{4}$ where they are fixed to the corresponding finger links (Fig. 9). Position of the finger is determined by its joint angles $\alpha_{i}, i=1, \cdots, 4$.

The model of friction force when tendon move over the pulley can be defined by the equation:

$$
F_{t}=F-F^{\prime}=F \frac{e^{\mu \alpha}-1}{e^{\mu \alpha}}
$$

where $\mu$ is Coulomb friction coefficient and $\alpha$ is the enclosed angle of tendon over the pulley.

Assuming that the friction coefficient (of tendons moving over the joint pulleys) is $\mu=0.14$ and choosing the compliance parameter, i.e. the spring stiffness to be $k=3.30 \frac{\mathrm{kg}}{\mathrm{cm}}$, the simulation experiments are made to validate the choice of the hand actuators and to check the critical tension of tendons against the ripping. Characteristic simulation results are given in Fig. 10. For the imposed banding of a finger (given by its joint angles) and by imposing of the external payload (force) acting to the distal phalanx of the finger in direction of the longitudinal axis, the joint payloads (torques) as well as the tendon dragging lengths, produced by the particular motors presented in Fig. 9, are showed in Fig. 10.

The tendon dragging lengths are non-linear functions of joint angles. The joint payloads are the consequence of finger payload (predominantly) and its motion. The calculated payloads do not overcome the technical limits of the RC motors applied.

Parallel with the mechanical design and system structural optimization the complementary research is conducted regarding acquisition of human grasping skill. In the following section, the emphasis was on exploring possibilities of our artificial hand to repeat and mimic human gestures. 
A. Rodić et al

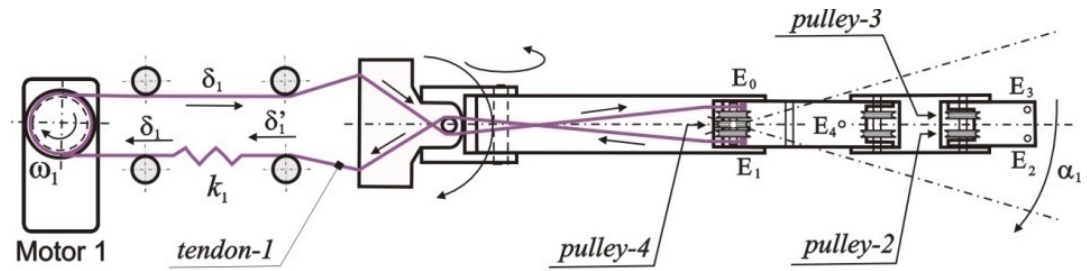

a)

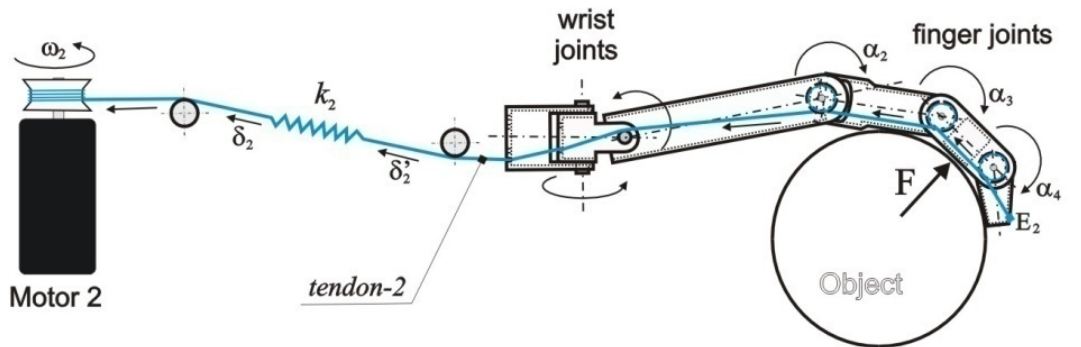

b)

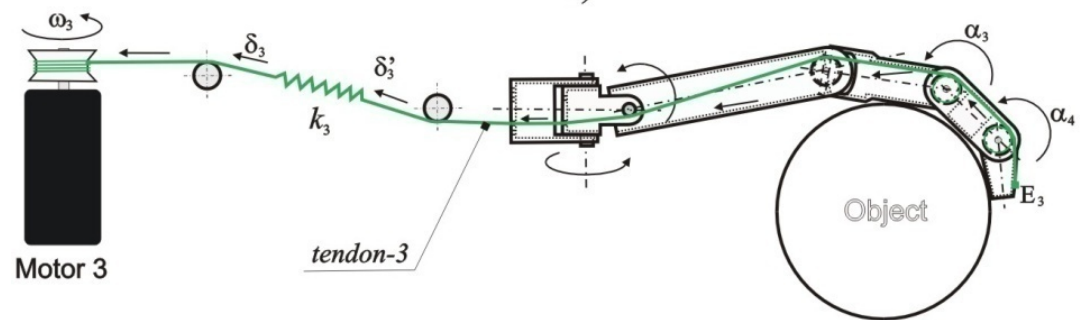

c)

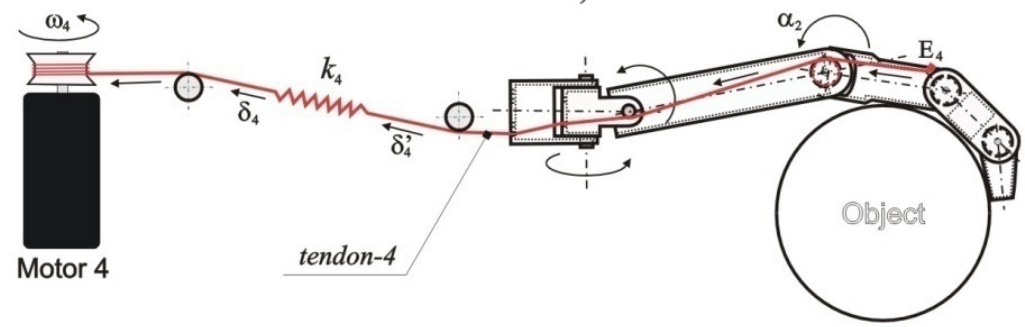

d)

Fig. 9 Principle of actuation of the 4 DoFs tendon-driven finger. (a) Swinging of the finger. (b) Finger bending, and (c/d) Two-stage springing of the finger. 

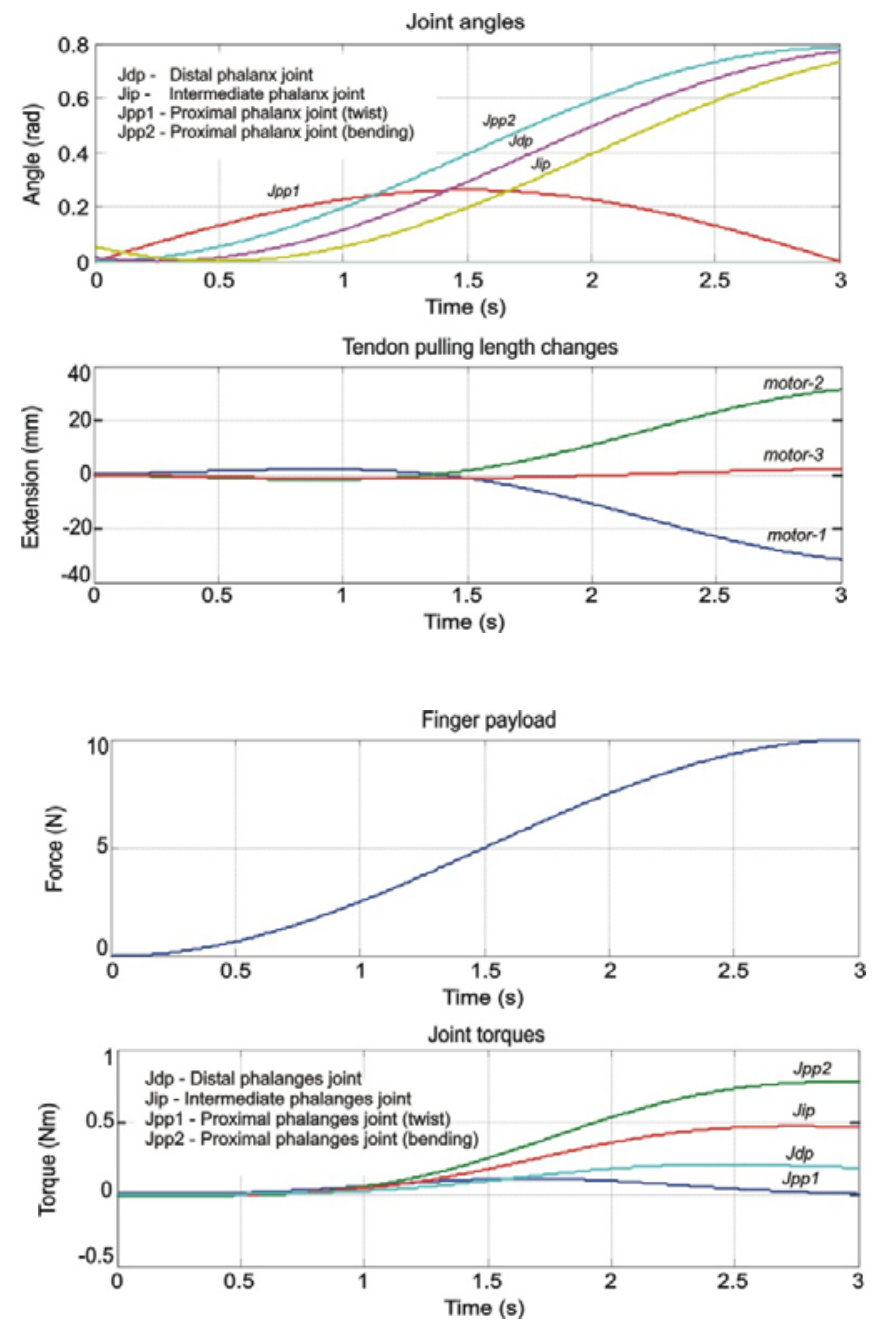

Fig. 10 Finger model simulation results: Joint angles; Drag lengths of the moving tendons; Imposed payload upon the distal phalanx; Corresponding joint payloads (torques).

\section{Acquisition of human grasping skill}

Development of techniques for capturing motion of human hands has been in focus of continuous interest of researchers and practitioners in human-centered robotics. This attention has been mainly due to two related although distinct tasks: 
trajectory programming and control of robotic hands (e.g., grasping trajectory) and human gesture recognition. For the purpose of this work, a detailed hand model has been adopted, incorporating 14 joints: 3 joints per each finger plus 2 thumb joints. The set of tracked points incorporated 22 points: finger/thumb tips (5), hand wrists (3), and 14 rotational joints (see Fig. 11).

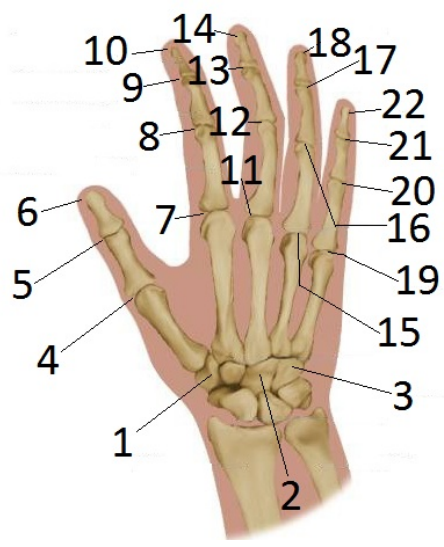

Fig. 11 Tracked hand points

Microsoft Kinect sensor [16] has been examined as a possible low cost alternative for capturing hand motion data. The device integrates an ordinary color camera with a depth sensor that is realized by combining an infrared dot pattern projector and infrared camera. Kinect was originally intended as input device in gaming applications. Yet, it has received a tremendous popularity in many other areas thanks to its low price combined with ease of programming and satisfactory performance. Additionally, the availability of several open source tools and the existence of an active user community make the integration of Kinect sensor fairly simple.

To improve Kinect accuracy, we used calibrated data. Calibration consisted in additional processing and adjusting data obtained from the sensor to compensate for nonlinearities in cameras and deviations in sensor parameters. The employed calibration model comprised intrinsic and extrinsic parameters of both color and infrared cameras [17] and additional parameters of Kinect depth-disparity model [18]. Model identification was realized as a two-step procedure, in which the first step comprised identification of camera parameters, whereas the second step was devoted to tuning of the depth model. The net result of the calibration is an augmented image in which each RGB pixel has associated 3D position. Extensive tests demonstrated that such calibrated sensor provided accuracy on the order from $3 \mathrm{~mm}$ for near objects to $15 \mathrm{~mm}$ for objects furthest on the sensor range.

Few typical hand motions were analyzed - cylindrical grasp, grasp of object from top position and lateral grasp. Hand and finger wrists had been previously 
labeled using special markers (Fig. 12). Joint position capturing was performed in two steps. First, positions of markers were detected on color image. Next, depth information and calibration parameters were used to obtain $3 \mathrm{D}$ positions of joints.

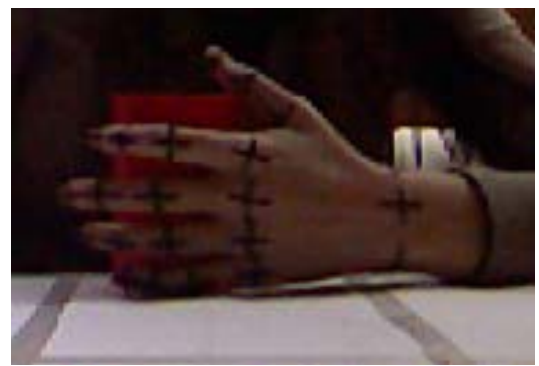

a)

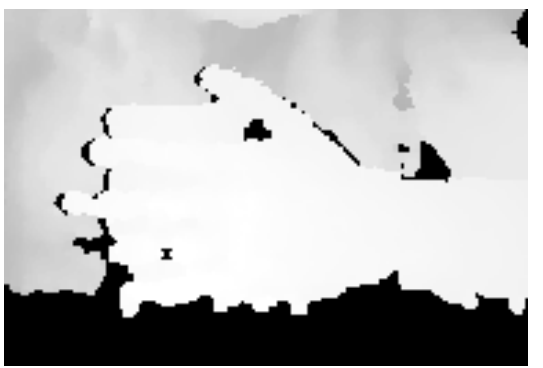

b)

Fig. 12 Sample augmented image for cylindrical grasp: a) color image, b) depth image.

A good insight into achieved accuracy could be obtained by analyzing measured lengths of finger segments. An illustration of variations of obtained lengths for a sample movement is shown in Fig. 13 and Table 1. It is seen that errors increase from root to distal segments.

Table 1. Measured lengths of the index finger segments

\begin{tabular}{ccccc}
\hline & \multicolumn{4}{c}{ INDEX FINGER } \\
\cline { 2 - 5 } & Metacarpal & $\begin{array}{c}\text { Proximal } \\
\text { phalanx }\end{array}$ & $\begin{array}{c}\text { Middle } \\
\text { phalanx }\end{array}$ & $\begin{array}{c}\text { Distal } \\
\text { phalanx }\end{array}$ \\
Average value $(\mathrm{cm})$ & 8.99 & 4.34 & 2.71 & 2.42 \\
Maximum deviation $(\mathrm{mm})$ & 1.89 & 2.57 & 5.93 & 6.02 \\
Average deviation $(\mathrm{mm})$ & 0.75 & 1.32 & 3.45 & 3.90 \\
Average deviation $(\%)$ & 0.84 & 3.04 & 12.70 & 16.11 \\
\hline
\end{tabular}

All measurements were done at the beginning of sensor field of view, on distance approximately 70 to $80 \mathrm{~cm}$ from Kinect sensor. Very good accuracy was achieved for joints located on a central part of hand (metacarpal and proximal phalanx joints). Length deviations for segments around these joints were on the order of $3 \%$ or less. 3D coordinates of joints located at the ends of fingers were characterized by much larger errors, up to $16 \%$. This result is a consequence of noise existing along boundaries between surfaces at different distance from sensor. As a result, due to large distance gradient, disparity values for these boundary pixels are generated imprecisely which directly leads to weak accuracy of obtained 3D positions. 


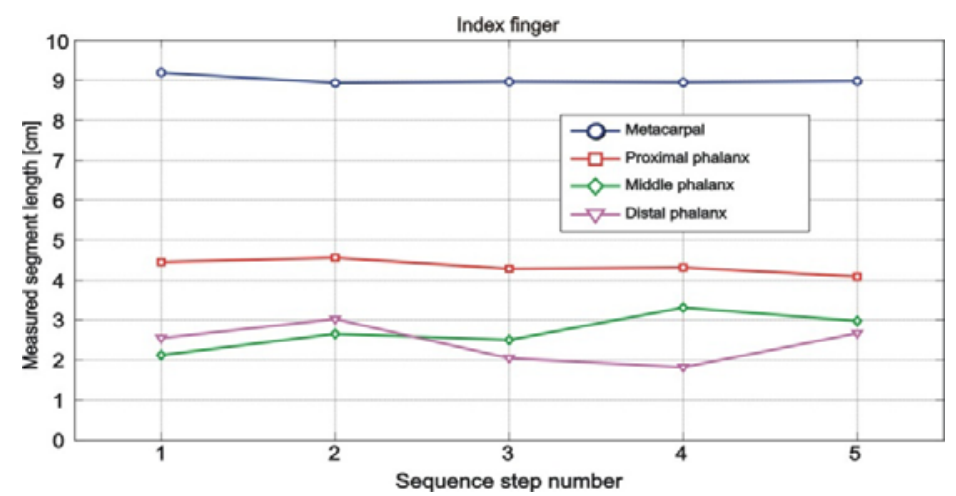

Fig. 13 Deviations in measured lengths of Index finger segments along sample movement.

This study demonstrated satisfactory level of accuracy when capturing positions of proximal joints and increased errors for distal joints. This leads to a conclusion that Kinect sensor could be successfully used for gesture recognition, whereas its usefulness would remain limited in applications where fine capture of trajectories is mandatory.

\section{Conclusions}

The next step in development of the Pupin hand regards to design of the forearm that should enable embedding of the hand actuators. Also, the associated electronics, sensorial system and microcontroller of the device is going to be added. Extensive testing of the real system are planned to be accomplished to validate hand feature characteristics. After that a robot hand of industrial quality is planned to be produced for different service applications.

Acknowledgments This work is funded by the Ministry of Education, Science and Technology Development of the Republic of Serbia under the contracts TR-35003, III-44008 and III-44004. Project is complementary supported by the SNSF IP SCOPES project under the grant ID: IZ74Z0_137361/1.

\section{References}

1. Shimon Nof, Handbook of Industrial Robots, John Wiley and Sons 1985

2 .M.S. Johannes, J. D. Bigelow, J. M. Burck, S. D. Harshbarger, M.V. Kozlowski and T. Van Doren: "An Overview of the Development Process for the Modular Prosthetic Limb", John Hopkins APL Techical Digest, Volume 30, Number 3, 2011, pp. 207-216.

3. Claudio Melchiori, Makoto Kaneko, Ch 15. Robot Hands, Handbook of Robotics, Siciliano, Bruno; Khatib, Oussama (Eds.) 2008, Springer. 
4. George A. Bekey, Rajko Tomovic, Ilija Zeljkovic, "Control Architecture for the Belgrade/USC Hand", in Dextrous Robot Hands, 1990, pp 136-149

5. Nency S. Pollard and Richards C. Gilbert: "Tendon Arrangement and Muscle Force Requirements for Humnalike Force Capabilities in a Robotic Finger"

6. M. Grebenstein, M. Chalon, W. Friedl, S. Haddadin, T. Wimbock, G. Hirzinfger and R. Siegwart: "The Hand of the DLR Hand Arm System. Designed for Interaction, The International Journal of Robotics Research 31(13), 2012, pp. 1531-1555.

7. H. Liu, K. Wu, P. Meusel, N. Seitz, G. Hirzinger (DLR), M.H. Jin, Y.W. Liu. S.W. Fan, T. Lan, Z.P. Chen (HIT): "Multisensory Five-Finger Dexterous Hand. The DLR7HIT Hand II", 2008 IEEE/RSJ International Conference on Intelligent Robots and Systems, Acropolis Convention center, Nice, France, Sept. 22-26, 2008, pp. 3692-3697.

8. J.H. Bae, S.W. Park, J.H. Park, M.H. Baeg, D. Kim and S.R. Oh: "Development of a Low Cost Anthropomorphic Robot hand with High Capability", 2012 IEEE/RSJ International Conference on Intelligent Robots and Systems, Vilamoura, Algarve, Portugal, Oct. 7-12, 2012, pp. 4776-4782.

9. Shadow Dexterous hand, (shadow Robot Ltd., London 2007), http://www.shadow.org.uc/

10. Jimmy W. Soto Martell, Giuseppina Gini: Robotic Hands: Design Review and Proposal of New Design Process, World Academy of Science, Engineering and Technology 26, 2007.

11. W. Friedl, H. Hoppner, F. Petit and G. Hirzinger: "Wrist and Forearm Rotation of the DLR Hand Arm System. Mechanical Design, Shape Analysis and Experimental Validation”, 2011 IEEE/RSJ International Conference on Robots and Systems, San Francisci, Ca, USA, Sept. 25-30, 2011, pp.1836-1842.

12. G. Palli, U. Scarcia, C. Melchiorri and G. Vassura: "Develepment of Robotic hands: The UB Hand Evolution", 2012 IEEE/RSJ International Conference on Intelligent Robots and Systems, Vilamoura, Algarve, Portugal, Oct. 7-12, 2012, pp. 5456-5457.

13. M. Grebenstein, M. Chalon, G. Hirzinger and R. Siegwart: “Antagonistically Driven Finger Design for the Anthropomorphic DLR Hand Arm system", 2010 IEEE/RAS International Conference on Humanoid Robots, Neshville, TN, USA, Dec. 6-8, 2011, pp 609-616.

14. On line: http://www.toyobo-global.com/seihin/dn/dyneema/seihin/tokutyou.htm

15. R.M. Murray, Z. Li, and S.S. Sastry. "A Mathematical Introduction to Robotic Manipulation". In: CRC Press, 1994. Chap. 6.4,pp. 293-298.

16. Kinect for windows, http://www.microsoft.com/en-us/kinectforwindows/ (accessed 26.03.2012)

17. Z. Zhang, "A flexible new technique for camera calibration," IEEE Trans. Pattern Anal. Mach. Intell., Vol. 22, No. 11, 2000, pp. 330-1334.

18. K. Khoshelham and S. O. Elberink, "Accuracy and Resolution of Kinect Depth Data for Indoor Mapping Applications," Sensors, Vol. 12, 2012, pp. 1437-1454. 